\title{
High prevalence of Plasmodium falciparum pfcrt K76T mutation in children with sickle cell disease at a tertiary hospital in north-western Tanzania
}

\author{
STELLA MONGELLA ${ }^{1}$, NIZAR ENWEJI ${ }^{2}$, NAIZIHIJWA MNONG'ONE ${ }^{1}$, MERCY MINDE ${ }^{1}$, ERASMUS \\ KAMUGISHA ${ }^{3^{*}}$ and GÖTE SWEDBERG ${ }^{2}$ \\ ${ }^{1}$ Department of Paediatrics, Catholic University of Health and Allied Sciences, Mwanza, Tanzania \\ ${ }^{2}$ Department of Medical Biochemistry and Microbiology, Uppsala University, Sweden \\ ${ }^{3}$ Department of Biochemistry, Catholic University of Health and Allied Sciences, Mwanza, Tanzania
}

\begin{abstract}
The high prevalence of sickle cell disease (SCD) and trait in Sub-Saharan Africa coincides with the distribution of Plasmodium falciparum malaria. Due to prolonged heavy use of chloroquine (CQ) as an antimalarial, drug resistance has developed. Many countries including Tanzania abandoned the use of CQ for uncomplicated malaria, except its use as prophylaxis in patients with sickle cell disease. This study investigated the prevalence of malaria in SCD patients and mutations associated with CQ resistance. Children diagnosed with sickle cell disease attending both outpatient clinic and those admitted at Bugando Medical Centre in north-western Tanzania were screened for malaria using thick blood smear. A dried blood spot on Whatman filter paper was also taken for polymerase chain reaction (PCR) and restriction fragment length polymorphism. Among 123 known patients with sickle cell disease, the prevalence of malaria by blood smear microscopy was $3.2 \%$ and by PCR was $13.8 \%$. The prevalence of K76T mutation among the patients was $81.3 \%$. The majority of the patients (72.4\%) were using chloroquine prophylaxis. In conclusion, the prevalence of malaria parasitaemia among children with sickle cell disease attending BMC is low (3.2\%) by microscopy but several children maintain sub patent infection detectable by PCR. The prevalence of chloroquine resistant $P$. falciparum in these children was higher than that previously seen in normal population in Tanzania. We recommend special attention to be paid to patients with sickle cell disease while studying the dynamics of drug resistant parasites.
\end{abstract}

Keywords: malaria, sickle cell disease, children, pcrt mutation, chemoprophylaxis, Tanzania

\section{Introduction}

The high prevalence of the sickle cell disease (SSD) and its trait in Sub-Saharan Africa coincides with the distribution of Plasmodium falciparum malaria (Serjeant et al., 2003). The unusually high frequency of sickle cell trait (HbAS) is maintained because carriers of that trait have lower rates of mortality from malaria infection. HbAS confers protection against severe malaria by limiting disease progression. Infections less often progress to the point at which either symptoms are evident (mild disease) or complications ensue (severe disease) (Williams et al., 2005). The occurrence of symptomatic malaria in individuals with homozygous sickle cell (HbSS) is however a major determinant of morbidity and mortality (Serjeant et al., 2003).

Previously, the World Health Organization (WHO) and many local national treatment guidelines advocated regular malaria chemoprophylaxis with chloroquine (CQ) among patients with sickle cell disease (MoHSW, 2006; WHO, 2006). The tremendous success of chloroquine and its heavy use through the decades eventually led to chloroquine resistance in Plasmodium falciparum (Wellems et al., 2001). Following the increase in drug resistance to CQ many countries including Tanzania changed treatment policy from CQ to sulphadoxine-pyrimethamine (SP) and later to artemisinin based combination therapies (ACTs). The changed treatment guidelines continued to advocate use of CQ for prophylaxis in SCD patients for some time (MoHSW, 2006). The recent national malaria treatment guideline clearly states that $C Q$ has no benefits but does not give an alternative drug for chemoprophylaxis in these patients (MoHSW, 2013). A study done in Uganda showed that children with sickle cell anaemia receiving weekly chloroquine were two times more likely to get malaria compared to those on monthly SP. This showed that SP was

\footnotetext{
* Correspondence: Erasmus Kamugisha; E-mail: erasmuskamugisha@yahoo.com
} 
more protective, as it reduced the prevalence of malaria by $50 \%$ compared to chloroquine (Nakiibuka et al., 2009).

Resistance against chloroquine in $P$. falciparum is associated with an amino acid change from lysine $(K)$ to threonine $(T)$ in codon 76 of the $P$. falciparum chloroquine resistance transporter gene ( $p f c r t$ ), which codes for a transporter protein in the parasite's digestive vacuole membrane (Wellems et al., 2001; Ginsburg et al., 2006). After discontinuation of CQ use, several studies in African countries have reported different prevalence of pfcrt K76T mutation. In some countries there is clear re-emergence of wild type strains coupled with CQ sensitivity (Kublin et al., 2003; Lauffer et al., 2006; Ginsburg et al., 2006; Frosch et al., 2011; Kamugisha et al., 2012; Mohamed et al., 2013; Malmberg et al., 2013). In other countries the level of CQ resistance is going down very slowly or is fixed (Frosch et al., 2011). Reasons for the difference in these countries are not clear but continued use of $\mathrm{CQ}$ for other purposes or combined treatment regimens for malaria have been proposed (Frosch et al., 2011).

Despite the high prevalence of SCD patients in malaria endemic areas, very little research has been done to assess the actual prevalence of $P$. falciparum and chloroquine resistance markers among this special population after deployment of CQ. This study was therefore, conducted to determine the prevalence of malarial parasitaemia and chloroquine resistant $P$. falciparum among children with sickle cell disease attending Bugando Medical Centre in northwestern Tanzania.

\section{Materials and Methods}

\section{Study site}

This was a hospital-based prospective cross-sectional study. The study was conducted from January to April 2012 at Bugando Medical Centre (BMC), a referral, research and teaching hospital, located in the North-western part of Tanzania. BMC serves a population of around 13 million people. There is a sickle cell disease outpatient clinic conducted once weekly whereby each patient is seen once a month. At the time of this study, there were about 225 children registered in this clinic.

\section{Study subjects}

All children with confirmed sickle cell disease under 12 years of age attending BMC inpatient and outpatient departments and the parent/guardian signed informed consent were eligible. Those with sickle cell disease and had history of antimalarial use other than chloroquine within two weeks prior to outpatient visit or admission, were excluded. Children with confirmed sickle cell disease by haemoglobin electrophoresis were screened as they reported into the paediatric outpatient clinic and inpatient wards. Data and clinical findings were collected using a standardized questionnaire after interviewing the parents/guardians and doing a thorough clinical examination to children.

\section{Laboratory analysis}

Malaria parasitaemia was confirmed with the use of Giemsa-stained thick blood films following standard methods. P. falciparum densities were assessed by counting the number of asexualstage parasites per 200 white blood cells and expressed as parasites per microliter of whole blood. DNA extraction from dried blood spots was done using Tris-EDTA as previously described (Bereczky et al., 2005). PCR and restriction fragment length polymorphism (RFLP) of the pfcrt gene was done using primers and conditions as previously established (Djimde et al., 2001). Full blood counts were performed with the use of haematology analyser CELL DYN 3700 (Abbott Laboratories, USA). 


\section{Ethical considerations}

The ethical clearance for conducting the study was granted from the Joint BMC-Catholic University of Health and Allied Sciences Publication and Ethical Committee. The signed informed consent was obtained from the respective parent or guardian before enrolment of the child. Treatment was done according to the hospital's paediatric department protocol for management of sickle cell disease and the Tanzania National Malaria Treatment Guidelines (MoHSW, 2006).

\section{Results}

A total of 123 children with SCD were enrolled of which 79 (64.2\%) were males. A majority of these children, 108 (87.8\%) were outpatients. The average age of diagnosis with SCD was 2 years and 4 months. The most common symptom on presentation was pain, which was reported in $14.6 \%$ of the children. The majority (68.3\%) of enrolled children had a palpable liver and only $29.3 \%$ had a palpable spleen. Hypoxemia was found in $21(17.1 \%)$ with a transcutaneous arterial oxygen saturation $\left(\mathrm{SaO}_{2}\right)$ of less than $90 \%$. The mean haemoglobin level was $7.2 \mathrm{~g} / \mathrm{dl}$ and $11.2 \%$ of those with severe anaemia had haemoglobin levels less than $5 \mathrm{~g} / \mathrm{dl}$ (Table 1 ).

Table 1: Clinical Presentation of Children with Sickle Cell Disease attending Bugando Medical Centre between January and April 2012

\begin{tabular}{ll}
\hline Clinical Presentation & Number $(\%)$ or Median (IQR) \\
\hline Splenomegaly & $36(29.3 \%)$ \\
Hepatomegaly & $84(68.3 \%)$ \\
Hypoxemia $\left(\mathrm{SaO}_{2}<90 \%\right.$ in Room Air) & $21(17.1 \%)$ \\
Haemoglobin level $(\mathrm{g} / \mathrm{dL})$ & $7.2(1.2-13.7)$ \\
Mild anaemia $(7-10 \mathrm{~g} / \mathrm{dL})$ & $64(55.2 \%)$ \\
Moderate anaemia $(5-6.9 \mathrm{~g} / \mathrm{dL})$ & $39(33.6 \%)$ \\
Severe anaemia $(<5 \mathrm{~g} / \mathrm{dL})$ & $13(11.2 \%)$ \\
Mean corpuscular volume $(\mathrm{fL})$ & $82.4(31.9-114)$ \\
Reticulocyte count $(\%)$ & $3.6(0.7-14.5)$ \\
White blood cell count $\left(\mathrm{x} 10^{9} / \mathrm{L}\right)$ & $13.0(2.78-38.2)$ \\
\hline
\end{tabular}

Of the children enrolled, $89(72.4 \%)$ were regularly taking chloroquine as antimalarial chemoprophylaxis, and $98.9 \%$ of them purchased the drug from local pharmacies and the remaining obtained them from dispensaries and health centres.

The prevalence of malaria by using blood smear microscopy was $3.3 \%$ (4/123) while when using PCR targeting a pfcrt gene segment, the prevalence of malaria was higher $13.8 \%(17 / 123)$ (Table 2). Restriction fragment length polymorphism was successful in 16 samples whereas one sample had not sufficient detectable digests by agarose gel electrophoresis. The prevalence of pfcrt mutations at position 76 was $81.3 \%$ (13/16) and the remaining showed the wild type allele. PCR picked more patients with malaria parasites that were not detected by smear microscopy giving it the sensitivity of $23.5 \%$ and specificity of $100 \%$ using blood smear microscopy as a gold standard test.

Table 2: Malarial Parasitaemia in children with Sickle Cell Disease

\begin{tabular}{lllll}
\hline & PCR Positive & PCR Negative & Sensitivity & Specificity \\
\hline BS Positive $(n=04)$ & 4 & 0 & & \\
BS Negative $(n=119)$ & 13 & 106 & $23.5 \%$ & $100 \%$ \\
Total & 17 & 106 & & \\
\hline
\end{tabular}




\section{Discussion}

The prevalence of malaria by blood smear microscopy in children with sickle cell disease (SCD) in this study was low. This finding is similar to the low malaria prevalence found recently in Dar es Salaam among sickle cell anaemia patients (Makani et al., 2010). Low prevalence of malaria among patients with HbSS has also been reported in other studies in Africa (Freidman et al., 1978; Aluoch, 1997; Cholera et al., 2008; Komba et al., 2009; Sadarangani et al., 2009). This is lower than the prevalence among primary school children in Mwanza, which was found to be $20 \%$ (Kamugisha et al., 2011). These cited studies that reported low prevalence used only blood smear microscopy. In this study when PCR was used the prevalence of malaria increased four-fold. This may be explained by existence of sub patent infections undetectable by microscopy. PCR has been reported to be more sensitive than microscopy in the detection of malaria parasites (Kilonzo et al., 2014). The use of recently developed diagnostic methods for malaria including rapid diagnostic tests and PCR are likely to readily identify and provide more reliable information on sub patent malaria infections in endemic areas.

The majority of children with SCD in this study reported to be on regular chloroquine chemoprophylaxis. This shows that SCD patients are still adhering to the treatment guidelines and therefore the new changes in malaria treatment policy will have to be communicated properly to these patients. In our study the prevalence of pfcrt K76T was high. The continued presence of resistance markers in this study may be explained by the continuing use of chloroquine for malaria chemoprophylaxis thus maintaining a selective pressure on these parasites. Similar findings have been reported from a study in Nigeria where the prevalence of pfcrt K76T among patients with SCD using chloroquine prophylaxis was higher among those with sickle cell anaemia compared than those with sickle cell trait (SCT) (Tatfeng et al., 2008). This prevalence of pfcrt K76T mutations is higher than those reported previously in parasites from Mwanza in Tanzania (Kamugisha et al., 2012a,b). These two studies included parasites from all patients and not a special group of patients with sickle cell disease. Similarly, higher prevalence of these mutants at pfcrt have been reported recently from elsewhere Tanzania (Ngasala et al., 2011; Malmberg et al., 2013; Mohamed et al., 2013). However, in these other Tanzanian studies the prevalence of patients with SCD was not stated.

Finding more mutant and hence resistant parasites in special groups of people on malaria prophylaxis has been reported previously (Tatfeng et al., 2008; Bertin et al., 2005). However, the findings did not attract more attention. Resistant parasites are important when trying to understand the dynamics of drug resistant parasites. They are continuously experiencing a selective pressure of CQ at a sub-therapeutic level, which is evidently selecting mutant parasites and maintain them at a higher level than in the normal population. SCD patients seem to be a special group that maintains sub patent level of mutant parasites that are not static and probably continues to be transmitted to other individuals. This group should be considered while studying the re-emergence of pfcrt wild type parasites especially in countries showing slower progress as reported by Frosch et al. (2011). Unpublished data (A. Kheir, unpubl) have revealed that parasites continue to change in patients during the dry season in Sudan when there is no transmission and are capable of being transmitted to other patients at the beginning of next transmission season.

The prevalence of malaria parasitaemia among children with sickle cell disease attending a tertiary hospital in north-western Tanzania is low by microscopy but several children maintain sub patent infection detectable by PCR. The prevalence of chloroquine resistant P. falciparum in these children was higher than that previously seen in normal populations in Tanzania. We recommend special attention to be paid to patients with sickle cell disease while studying the dynamics of drug resistant parasites and the treatment guidelines to determine options for malaria chemoprophylaxis in this special group.

\section{References}


Aluoch, J.R. (1997) Higher resistance to Plasmodium falciparum infection in patients with homozygous sickle cell disease in western Kenya. Tropical Medicine and International Health 2, 568-71.

Ashley-Koch, A., Yang, Q. \& Olney, R.S. (2000) Sickle hemoglobin (HbS) allele and sickle cell disease: A HuGE review. American Journal of Epidemiology 151, 839-845.

Awotua-Efebo, O., Alikor, E.A. \& Nkanginieme, K.E. (2004) Malaria parasite density and splenic status by ultrasonography in stable sickle-cell anaemia (HbSS) children. Nigerian Journal of Medicine 13, 40-43.

Bereczky, S., Martensson, A., Gil, J.P. \& Farnert, A. (2005) Rapid DNA extraction from archive blood spots on filter paper for genotyping of Plasmodium falciparum. American Journal of Tropical Medicine and Hygiene 72, 249-251.

Bertin, G., Ndam, N.T., Jafari-Guemouri, S., Fievet, N., Renart, E., Sow, S., Hesran, J.L. \& Deloron, P. (2005) High prevalence of Plasmodium falciparum pfcrt K76T mutation in pregnant women taking chloroquine prophylaxis in Senegal. JAC 55, 788-791

Cholera, R., Brittain, N.J., Gillrie, M.R., Lopera-Mesa, T.M., Diakite, S.A.S., Arie, T., Krause, M.A., Guindo, A., Tubman, A., Fujioka, H., Diallo, D.A., Doumbo, O.K., Ho, M., Wellems, T.E. \& Fairhurst, R.M. (2008) Impaired cytoadherence of Plasmodium falciparum-infected erythrocytes containing sickle hemoglobin. PNAS 105, 991-6.

Djimde, A., Doumbo, O.K., Cortese, J.F., Kayentao, K., Doumbo, S., Diourte, Y., Dicko, A., Su, X., Nomura, T., Fidock, D.A., Wellems, T.E. \& Plowe, C.V. (2001) A molecular marker for chloroquine-resistant falciparum malaria. New England Journal of Medicine 344, 257-263.

Freidman, M. (1978) Erythrocytic mechanism of sickle cell resistance to malaria. Proceedings of the National Academy of Science of the United States of America 75, 1994-7.

Frosch, A.E.P., Venkatesan, M. \& Laufer, M.K. (2011). Patterns of chloroquine use and resistance in Sub-Saharan Africa: a systematic review of household survey and molecular data. Malaria Journal 10:116.

Ginsburg, H. (2005) Should chloroquine be laid to rest? Acta Tropica 96,16-23.

Kamugisha, E., Bujila, I., Lahdo, M., Pello-Esso, S., Minde, M., Kongola, G., Naiwumbwe, H., Kiwuwa, S., Kaddumukasa, M., Kironde, F. \& Swedberg G. (2012a) Large differences in prevalence of pfcrt and pfmdr1 mutations between Mwanza, Tanzania and Iganga, Uganda-A reflection of differences in policies regarding withdrawal of chloroquine? Acta Tropica 121, 148- 151.

Kamugisha, E., Jing, S., Minde, M., Kataraihya, J., Kongola, G., Kironde, F. \& Swedberg, G. (2012b) Efficacy of artemether-lumefantrine in treatment of malaria among under-fives and prevalence of drug resistance markers in Igombe-Mwanza, north-western Tanzania. Malaria Journal 11:58.

Kamugisha, E., Manyama, M., Rambau, P., Mazigo, H.D., Mshana, S.E. \& Masesa, Z. (2011) Prevalence of sickle cell, malaria and glucose-6-phosphate dehydrogenase deficiency among primary school children in Nyamagana District, Mwanza-Tanzania. Tanzania Medical Journal 25 (1).

Kilonzo, S.B., Kamugisha, E., Downs, J.A., Kataraihya, J., Onesmo, R., Mheta, K., Jeong, J.M., Verweij, J.J., Fitzgerald, D.W. \& Peck, R.N. (2014) Malaria among adult inpatients in two Tanzanian referral hospitals: a prospective study. Acta Tropica 134,95-100

Komba, A.N., Makani, J., Sadaragran, M., Ajala-Agbo, T., Berkley, J.A, Newton, C.R.J.C., Marsh, K. \& Williams T.N. (2009) Malaria as a cause of morbidity and mortality in children with homozygous sickle cell disease on the coast of Kenya. Clinical Infectious Diseases 49, 21622.

Kublin, J.G., Cortese, J.F., Njunju, E.M., Mukadam, R.A., Wirima, J.J., Kazembe, P.N., Djimde, A.A., Kouriba, B., Taylor, T.E. \& Plowe, C.V. (2003) Reemergence of chloroquine-sensitive Plasmodium falciparum malaria after cessation of chloroquine use in Malawi. Journal of Infectious Diseases 187, 1870-1875. 
Makani, J., Komba, A.N., Cox, S.E., Oruo, J., Mwamtemi, K., Kitundu, J., Magesa, P., Rwezaula, S., Meda, E., Mgaya, J., Pallangyo, K., Okiro, E., Muturi, D., Newton, C.R., Fegan, G., Marsh, K. \& Williams, T.N. (2010) Malaria in patients with sickle cell anaemia: burden, risk factors and outcome at the outpatient clinic and during hospitalisation. Blood 115, 215-20.

Malmberg, M., Ngasala, B., Ferreira, P.E., Larson, E., Jovel, I., Hjalmarsson, A., Petzold, M., Premji, Z., Gil, J.P., Bjorkman, A. \& Martenson, A. (2013) Temporal trends of molecular markers associated with artemether-lumefantrine tolerance/resistance in Bagamoyo district, Tanzania. Malaria Journal 12:103.

Mohamed, A., Ndaro, A., Kalinga, A., Manjurano, A., Mosha, J.F., Mosha, D.F., Zwetselaar, M., Koenderink, J.B., Mosha, F.W., Alifrangis, M., Reyburn, H., Roper, C. \& Kavishe R.A. (2013) Trends in chloroquine resistance marker, pcrt-K76T mutation ten years after chloroquine withdrawal in Tanzania. Malaria Journal 12:415

MoHSW (2006) National Guidelines for Malaria Diagnosis and Treatment. Malaria Control Series 11. Ministry of Health and Social Welfare, United Republic of Tanzania.

MoHSW (2013) National Guidelines for Malaria Diagnosis and Treatment. Malaria Control Series 25. Ministry of Health and Social Welfare, United Republic of Tanzania.

Ngasala, B.E., Malmberg, M., Carlsson, A.M., Ferreira, P.E., Petzold, M.G., Blessborn, D., Bergqvist, Y., Gil, J.P., Premji, Z., Björkman, A. \& Mårtensson, A. (2011) Efficacy and effectiveness of artemether-lumefantrine after initial and repeated treatment in children $<5$ years of age with acute uncomplicated Plasmodium falciparum malaria in rural Tanzania: a randomized trial. Clinical Infectious Diseases 52, 873-882

Sadarangani, M., Makani, J., Komba, A.N., Ajala-Agbo, T., Newton, C.R., Marsh, K. \& Williams, T.N. (2009) An observational study of children with sickle cell disease in Kilifi, Kenya. British Journal of Haematology 146, 675-682.

Serjeant, G.R. \& Ndugwa, C.M. (2003) Sickle cell disease in Uganda: a time for action. East African Medical Journal 80, 384-387.

Tatfeng, Y.M., Agbonlahor, D.E., Tchounga, K.S., Omolu, P.I., Okodua, M., Yah, C.S. \& Adeolu, A. (2008) Chloroquine prophylaxis associated with high prevalence of Plasmodium falciparum pfcrt K76T mutation in people with sickle-cell disease in Benin City, Nigeria. Journal of Vector Borne Diseases 45, 51-55

Wellems, T.E. \& Plowe, C.V. (2001) Chloroquine-Resistant Malaria. The Journal of Infectious Diseases 184, 770-6.

WHO (2006) Sickle-cell anaemia. $59^{\text {th }}$ World Health Assembly. Accessed at: http://apps.who.int

Williams, T.N., Mwangi, T.W., Wambua, S., Alexander, N.D., Kortok, M., Snow, R.W. \& Marsh, K. (2005) Sickle cell trait and the risk of Plasmodium falciparum malaria and other childhood diseases. Journal of Infectious Diseases 192, 178-86. 